\title{
The structure of prime sums
}

\author{
J. V. Leyendekkers ${ }^{1}$ and A. G. Shannon ${ }^{2}$ \\ ${ }^{1}$ Faculty of Science, The University of Sydney, NSW 2006, Australia \\ e-mail: jeanvaldek@gmail.com \\ ${ }^{2}$ Emeritus Professor, University of Technology Sydney, NSW 2007, Australia, and \\ Warrane College, The University of New South Wales, NSW 2033, Australia \\ e-mails: tshannon38@gmail.com, t. shannon@warrane.unsw.edu.au
}

\begin{abstract}
The development of prime sequences has allowed the detailed structure of prime sums to be made. Such structures help to explain why some sums yield primes while others give composites. The application of right-end-digit (modulo 10) structure permits analysis independent of the size of the integers being examined.
\end{abstract}

Keywords: Prime numbers, Composite numbers, Right-end-digits, Modulus.

2010 Mathematics Subject Classification: 11A07, 11A51, 11B37.

\section{Introduction}

Prime sums feature in a number of conjectures, such as that all odd integers greater than 5 can be formed from the sum of three primes [cf. 1]. Recent studies of prime sequences [2, 3, 4] allow the structure of the sums to be analysed so that the primality of the sum can be explained.

This is done by considering the integers in the form $n R$ where $R$ is the right-end-digit and $n$ the remaining digits. For example, for $N=20779, n=2077$ and $R=9$. The work is predicated on the only REDs for primes are 1, 3, 7, 9, though not every integer with a RED of 1 , or 3, or 7, or 9 , is necessarily prime.

The $n$ values can be classified for our purposes into three sequences $\{3 t\},\{3 t+1\},\{3 t+2\}$. Integers can never be prime when

- $n \in\{3 t\}, R \in\{3,9\}$, or

- $n \in\{3 t+2\}, R \in\{1,7\}$.

Furthermore, an integer can also have an $n$ value which prevents the formation of a prime for certain $R$ values if $n$ belongs to an imbedded sequence of the form $\{a+b j\}$, in which $a$ has the form of the main sequence and $3 \mid b$ (Table 1). 


\begin{tabular}{|c|c|}
\hline$R=1$ & $(a, b)$ \\
\hline$n \in\{3 t\}$ & $(3,93)[n>3],(9,21),(9,39),(12,33),(36,57),(39,51),(84,87)$ \\
\hline$n \in\{3 t+1\}$ & $(1,66)[n>1],(16,21),(16,69),(22,39),(22,51),(34,66)$ \\
\hline$n \in\{3 t+2\}$ & No primes formed \\
\hline$R=3$ & $(a, b)$ \\
\hline$n \in\{3 t\}$ & No primes formed \\
\hline$n \in\{3 t+1\}$ & $(1,39),(13,21),(13,57),(25,33),(25,69),(49,51)$ \\
\hline$n \in\{3 t+2\}$ & $(2,69)[n>2],(14,33),(14,39),(20,21),(32,51),(32,57)$ \\
\hline$R=7$ & $(a, b)$ \\
\hline$n \in\{3 t\}$ & $(0,21),(18,33),(18,51),(24,39),(24,57),(66,69)$ \\
\hline$n \in\{3 t+1\}$ & $(1,51)[n>1],(7,21),(7,66),(37,39),(43,57)$ \\
\hline$n \in\{3 t+2\}$ & No primes formed \\
\hline$R=9$ & $(a, b)$ \\
\hline$n \in\{3 t\}$ & No primes formed \\
\hline$n \in\{3 t+1\}$ & $(1.57)[n>1],(4,21),(16,39),(28,51),(31,33),(52,69),(64,231),(79$, \\
& $102)$ \\
\hline$n \in\{3 t+2\}$ & $(11,21),(11,51),(20,33),(20,57),(29,39),(29,69),(32,42),(89,93)$ \\
\hline
\end{tabular}

Table 1. Imbedded sequences which block prime formation for particular REDs

\section{Some prime sums}

The $n$ value of the sum of the REDs depends on the number of primes being added and the value of the RED; for instance, the sum of 9, 9, 9 is 27 with $n=2$ and $R=7$, whereas the sum of seven REDs of 9 would yield an $n$ of 6 and an $R$ of 3 . To illustrate the detailed structure we use threeprime sums and the various $R$ sets which give a sum with a particular $R$ (Table 2).

\begin{tabular}{|c|c|c|c|c|c|c|c|}
\hline & \multicolumn{6}{|c|}{ Some prime triples' REDs whose sums yield the $R$} & \multirow{3}{*}{$R$ sum } \\
\hline \multirow{2}{*}{$n_{R} \in$} & \multirow{2}{*}{\multicolumn{2}{|c|}{$\begin{array}{c}\{3 t\} \\
0\end{array}$}} & \multirow{2}{*}{\multicolumn{2}{|c|}{$\begin{array}{c}\{3 t+1\} \\
1\end{array}$}} & \multirow{2}{*}{\multicolumn{2}{|c|}{$\begin{array}{c}\{3 t+2\} \\
2\end{array}$}} & \\
\hline & & & & & & & \\
\hline & & & $1,1,9$ & $1,3,7$ & $3,9,9$ & $7,7,7$ & 1 \\
\hline & $1,1,1$ & & $1,3,9$ & $3,3,7$ & $7,7,9$ & & 3 \\
\hline & $1,3,3$ & & $1,7,9$ & $3,7,7$ & $9,9,9$ & & 7 \\
\hline & $1,1,7$ & $3,3,3$ & $3,9,7$ & $1,9,9$ & & & 9 \\
\hline
\end{tabular}

Table 2. REDs of some prime sums

The detailed structure of the sums of three primes, chosen at random, are listed in Tables 3, 4,5 , and 6 . The reasons for the primality of the sums are shown in the last column. Primes are formed when the $n$ value of the sum is compatible with the main sequence and/or the imbedded sequence. In these tables,

$$
t_{0}=\left\lfloor\frac{n}{3}\right\rfloor
$$

so that

$$
n=3 t_{0}+a, a=0,1 \text { or } 2 .
$$




\begin{tabular}{|c|c|c|c|c|c|c|c|c|c|c|}
\hline Set & $p_{1}$ & $p_{2}$ & $p_{3}$ & $n_{1} \in$ & $n_{2} \in$ & $n_{3} \in$ & $t_{1}$ & $t_{2}$ & $t_{3}$ & $\Sigma \boldsymbol{n}=$ \\
\hline $\begin{array}{c}1,1,9 \\
(11)\end{array}$ & 41 & 71 & 79 & $\left\{3 t_{1}+1\right\}$ & $\left\{3 t_{2}+1\right\}$ & $\left\{3 t_{3}+1\right\}$ & 1 & 2 & 2 & $3((\Sigma \mathrm{t})+1)=18$ \\
\hline $\begin{array}{c}1,1,9 \\
(11)\end{array}$ & 41 & 71 & 89 & $\left\{3 t_{1}+1\right\}$ & $\left\{3 t_{2}+1\right\}$ & $\left\{3 t_{3}+2\right\}$ & 1 & 2 & 2 & $3(\Sigma \mathrm{t}+1)+1=$ \\
\hline $\begin{array}{c}1,1,9 \\
(11)\end{array}$ & 31 & 61 & 89 & $\left\{3 t_{1}\right\}$ & $\left\{3 t_{2}\right\}$ & $\left\{3 t_{3}+2\right\}$ & 1 & 2 & 2 & $3(\Sigma \mathrm{t})+2=17$ \\
\hline $\begin{array}{c}1,1,9 \\
(11)\end{array}$ & 61 & 131 & 179 & $\left\{3 t_{1}\right\}$ & $\{3 t+1\}$ & $\left\{3 t_{3}+2\right\}$ & 2 & 4 & 5 & $3((\Sigma \mathrm{t})+1)=36$ \\
\hline $\begin{array}{c}1,3,7 \\
(11)\end{array}$ & 211 & 653 & 157 & $\left\{3 t_{1}\right\}$ & $\left\{3 t_{2}+2\right\}$ & $\left\{3 t_{3}\right\}$ & 7 & 21 & 5 & $3(\Sigma \mathrm{t})+2=101$ \\
\hline \begin{tabular}{c}
$1,3,7(11)$ \\
\hline
\end{tabular} & 252 & 283 & 257 & $\left\{3 t_{1}+1\right\}$ & $\left\{3 t_{2}+1\right\}$ & $\left\{3 t_{3}+1\right\}$ & 8 & 9 & 8 & $3((\Sigma \mathrm{t})+1)=78$ \\
\hline
\end{tabular}

Table 3(a). Structure of $\Sigma n$ in three prime sum with $R=1$

\begin{tabular}{|c|c|c|c|c|c|c|c|c|}
\hline $\begin{array}{l}\text { Set, } \\
\Sigma \text { Set }\end{array}$ & $p_{1}$ & $p_{2}$ & $p_{3}$ & $n_{R}$ & $\begin{array}{c}n= \\
n_{R}+\Sigma n\end{array}$ & $a$ & $t_{0}$ & Comments \\
\hline $\begin{array}{l}1,1,9 \\
(11)\end{array}$ & 41 & 71 & 79 & 1 & 19 & 1 & 6 & 191 (p); sequences compatible \\
\hline $\begin{array}{l}1,1,9 \\
(11)\end{array}$ & 41 & 71 & 89 & 1 & 20 & 2 & 6 & $\begin{array}{l}201(\mathrm{c}) \text {; when } n=3 t+2, R=1 \text { never } \\
\text { prime }\end{array}$ \\
\hline $\begin{array}{c}1,1,9 \\
(11)\end{array}$ & 31 & 61 & 89 & 1 & 18 & 0 & 6 & $181(\mathrm{p})$; sequences compatible \\
\hline $\begin{array}{c}1,1,9 \\
(11)\end{array}$ & 61 & 131 & 179 & 1 & 37 & 1 & 12 & $\begin{array}{l}371 \text { (c); main sequence compatible, but } \\
\text { imbedded sequence } n=16+21 j, j=1 \\
\text { prevents } R=1 \text { forming a prime (Table } 1)\end{array}$ \\
\hline $\begin{array}{c}1,3,7 \\
(11)\end{array}$ & 211 & 653 & 157 & 1 & 102 & 0 & 34 & $1021(\mathrm{p})$; sequences compatible \\
\hline $\begin{array}{c}1,3,7 \\
(11)\end{array}$ & 252 & 283 & 257 & 1 & 79 & 1 & 26 & $\begin{array}{l}791 \text { (c); main sequence compatible; } \\
\text { imbedded sequence } n=16+21 j, j=3 \\
\text { prevents } R=1 \text { forming a prime }\end{array}$ \\
\hline
\end{tabular}

Table 3(b). Structure of three prime sum with $R=1$

\begin{tabular}{|c|c|c|c|c|c|c|c|c|c|c|}
\hline Set & $p_{1}$ & $p_{2}$ & $p_{3}$ & $n_{1} \in$ & $n_{2} \in$ & $n_{3} \in$ & $t_{1}$ & $t_{2}$ & $t_{3}$ & $\Sigma \boldsymbol{n}=$ \\
\hline $3,3,7$ & 523 & 1783 & 2857 & $\left\{3 t_{1}+1\right\}$ & $\left\{3 t_{2}+1\right\}$ & $\left\{3 t_{3}\right\}$ & 17 & 59 & 95 & $3(\Sigma \mathrm{t})+2=515$ \\
\hline $7,7,9$ & 5197 & 2917 & 1609 & $\left\{3 t_{1}\right\}$ & $\left\{3 t_{2}\right\}$ & $\left\{3 t_{3}+1\right\}$ & 173 & 97 & 53 & $3(\Sigma \mathrm{t})+1=970$ \\
\hline $7,7,9$ & 337 & 1217 & 2579 & $\left\{3 t_{1}\right\}$ & $\left\{3 t_{2}+1\right\}$ & $\left\{3 t_{3}+2\right\}$ & 11 & 40 & 85 & $3(\Sigma \mathrm{t}+1)=411$ \\
\hline $1,1,1$ & 5981 & 641 & 2621 & $\left\{3 t_{1}+1\right\}$ & $\left\{3 t_{2}+1\right\}$ & $\left\{3 t_{3}+1\right\}$ & 199 & 21 & 87 & $3(\Sigma \mathrm{t}+1)=924$ \\
\hline $1,1,1$ & 5981 & 601 & 2621 & $\left\{3 t_{1}+1\right\}$ & $\left\{3 t_{2}\right\}$ & $\left\{3 t_{3}+1\right\}$ & 199 & 20 & 87 & $3(\Sigma \mathrm{t})+2=920$ \\
\hline
\end{tabular}

Table 4(a). Structure of $\Sigma n$ in three prime sum with $R=3$ 


\begin{tabular}{|c|c|c|c|c|c|c|c|l|}
\hline $\begin{array}{c}\text { Set, } \\
\boldsymbol{\Sigma} \text { Set }\end{array}$ & $p_{1}$ & $p_{2}$ & $p_{3}$ & $n_{R}$ & $\begin{array}{c}\boldsymbol{n}= \\
\boldsymbol{n}_{\boldsymbol{R}}+\boldsymbol{\Sigma} \boldsymbol{n}\end{array}$ & $\boldsymbol{a}$ & $\boldsymbol{t}_{\boldsymbol{0}}$ & \multicolumn{1}{|c|}{ Comments } \\
\hline $\begin{array}{c}3,3,7 \\
(11)\end{array}$ & 523 & 1783 & 2857 & 1 & 516 & 0 & 172 & $\begin{array}{l}5163(\mathrm{c}) ; \text { main sequence } \\
\text { incompatible }\end{array}$ \\
\hline $\begin{array}{c}7,7,9 \\
(23)\end{array}$ & 5197 & 2917 & 1609 & 2 & 972 & 0 & 324 & $\begin{array}{l}9723(\mathrm{c}) ; \text { main sequence } \\
\text { incompatible }\end{array}$ \\
\hline $\begin{array}{c}7,7,9 \\
(23)\end{array}$ & 337 & 1217 & 2579 & 2 & 413 & 2 & 137 & $\begin{array}{l}4133(\mathrm{p}) ; \text { main sequence } \\
\text { compatible; imbedded sequence } \\
n=14+21 j, j=19\end{array}$ \\
\hline $\begin{array}{c}1,1,1 \\
(03)\end{array}$ & 5981 & 641 & 2621 & 0 & 924 & 0 & 308 & $\begin{array}{l}9243(\mathrm{c}) ; \text { main sequence } \\
\text { incompatible }\end{array}$ \\
\hline $\begin{array}{c}1,1,1 \\
(03)\end{array}$ & 5981 & 601 & 2621 & 0 & 920 & 2 & 306 & $\begin{array}{l}9203(\mathrm{p}) ; \text { main sequence } \\
\text { compatible; imbedded sequence } \\
n=17+21 j, j=43\end{array}$ \\
\hline
\end{tabular}

Table 4(b). Structure of three prime sum with $R=3$

\begin{tabular}{|c|c|c|c|c|c|c|c|c|c|c|}
\hline Set & $p_{1}$ & $p_{2}$ & $p_{3}$ & $n_{1} \in$ & $n_{2} \in$ & $n_{3} \in$ & $t_{1}$ & $t_{2}$ & $t_{3}$ & $\Sigma n=$ \\
\hline $9,9,9$ & 2039 & 929 & 2969 & $\left\{3 t_{1}+2\right\}$ & $\left\{3 t_{2}+2\right\}$ & $\left\{3 t_{3}+2\right\}$ & 67 & 30 & 98 & $3((\Sigma \mathrm{t})+2)=591$ \\
\hline $1,3,3$ & 571 & 2143 & 5153 & $\left\{3 t_{1}\right\}$ & $\left\{3 t_{2}+1\right\}$ & $\left\{3 t_{3}+2\right\}$ & 19 & 71 & 171 & $3((\Sigma \mathrm{t})+1)=786$ \\
\hline $1,7,9$ & 3461 & 4027 & 1319 & $\left\{3 t_{1}+1\right\}$ & $\left\{3 t_{2}\right\}$ & $\left\{3 t_{3}+2\right\}$ & 115 & 134 & 43 & $3((\Sigma \mathrm{t})+1)=879$ \\
\hline $7,7,3$ & 4177 & 2897 & 2293 & $\left\{3 t_{1}\right\}$ & $\left\{3 t_{2}+1\right\}$ & $\left\{3 t_{3}+1\right\}$ & 139 & 96 & 76 & $3(\Sigma \mathrm{t})+2=935$ \\
\hline
\end{tabular}

Table 5(a). Structure of $\Sigma n$ in three prime sum with $R=7$

\begin{tabular}{|c|c|c|c|c|c|c|c|l|}
\hline $\begin{array}{c}\text { Set, } \\
\boldsymbol{\Sigma} \text { Set }\end{array}$ & $p_{1}$ & $p_{2}$ & $p_{3}$ & $n_{R}$ & $\boldsymbol{n}$ & $\boldsymbol{a}$ & $\boldsymbol{t}_{\mathbf{0}}$ & \multicolumn{1}{|c|}{ Comments } \\
\hline $\begin{array}{c}9,9,9 \\
(27)\end{array}$ & 2039 & 929 & 2969 & 2 & 593 & 2 & 197 & $\begin{array}{l}5937(\mathrm{c}) ; \text { Main sequence prevents primes } \\
\text { as } R=1 \text { or 7 yields a composite } \\
\text { (Table 1) }\end{array}$ \\
\hline $\begin{array}{c}1,3,3 \\
(07)\end{array}$ & 571 & 2143 & 5153 & 0 & 786 & 0 & 262 & $\begin{array}{l}7867(\mathrm{p}) ; \text { main sequence compatible; and } \\
\text { imbedded sequence } n=9+21 j, \\
j=37\end{array}$ \\
\hline $\begin{array}{c}1,7,9 \\
(17)\end{array}$ & 3461 & 4027 & 1319 & 1 & 880 & 1 & 880 & $\begin{array}{l}8807(\mathrm{p}) ; \text { main sequence compatible; and } \\
\text { imbedded sequence } n=19+21 j, \\
j=41\end{array}$ \\
\hline $\begin{array}{c}7,7,3 \\
(17)\end{array}$ & 4177 & 2897 & 2293 & 1 & 936 & 0 & 936 & $\begin{array}{l}9367 \text { (c); main sequence compatible; } \\
\text { im-bedded sequence } n=18+51 j, \\
j=18 \text { (in Table 1) }\end{array}$ \\
\hline
\end{tabular}

Table 5(b). Structure of three prime sum with $R=7$ 


\begin{tabular}{|c|c|c|c|c|c|c|c|c|c|c|}
\hline $\begin{array}{c}\text { Set, } \\
\Sigma \text { Set }\end{array}$ & $p_{1}$ & $p_{2}$ & $p_{3}$ & $n_{1} \in$ & $n_{2} \in$ & $n_{3} \in$ & $t_{1}$ & $t_{2}$ & $t_{3}$ & $\Sigma n=$ \\
\hline $1,1,7$ & 3701 & 5281 & 5987 & $\left\{3 t_{1}+1\right\}$ & $\left\{3 t_{2}\right\}$ & $\left\{3 t_{3}+1\right\}$ & 123 & 176 & 199 & $3(\Sigma \mathrm{t})+2=1496$ \\
\hline $9,9,1$ & 166 & 97 & 130 & $\left\{3 t_{1}+1\right\}$ & $\left\{3 t_{2}+2\right\}$ & $\left\{3 t_{3}+1\right\}$ & 166 & 97 & 130 & $3((\Sigma \mathrm{t})+1)+1=1183$ \\
\hline $3,9,7$ & 3203 & 5209 & 5987 & $\left\{3 t_{1}+2\right\}$ & $\left\{3 t_{2}+1\right\}$ & $\left\{3 t_{3}+1\right\}$ & 106 & 173 & 199 & $3((\Sigma \mathrm{t})+1)+1=1438$ \\
\hline $3,3,3$ & 7393 & 6043 & 4243 & $\left\{3 t_{1}+1\right\}$ & $\left\{3 t_{2}+1\right\}$ & $\left\{3 t_{3}+1\right\}$ & 246 & 201 & 141 & $3((\Sigma \mathrm{t})+1)=1767$ \\
\hline $3,3,3$ & 1783 & 2843 & 3643 & $\left\{3 t_{1}+1\right\}$ & $\left\{3 t_{1}+2\right\}$ & $\left\{3 t_{3}+1\right\}$ & 59 & 94 & 121 & $3((\Sigma \mathrm{t})+1)+1=826$ \\
\hline
\end{tabular}

Table 6(a). Structure of $\Sigma n$ in three prime sum with $R=9$

\begin{tabular}{|c|c|c|c|c|c|c|c|c|}
\hline $\begin{array}{l}\text { Set, } \\
\Sigma \text { Set }\end{array}$ & $p_{1}$ & $p_{2}$ & $p_{3}$ & $n_{R}$ & $n$ & $a$ & $t_{0}$ & Comments \\
\hline $\begin{array}{c}1,1,7 \\
(09)\end{array}$ & 3701 & 5281 & 5987 & 0 & 1496 & 2 & 498 & $14969(\mathrm{p})$; sequences compatible \\
\hline $\begin{array}{l}9,9,1 \\
(19)\end{array}$ & 166 & 97 & 130 & 1 & 1184 & 2 & 394 & $\begin{array}{l}11849(\mathrm{c}) \text {; main sequence compatible; } \\
\text { but not imbedded sequence } \\
n=11+51 j, j=23 \text { (Table } 1)\end{array}$ \\
\hline $\begin{array}{c}3,9,7 \\
(19)\end{array}$ & 3203 & 5209 & 5987 & 1 & 1439 & 2 & 479 & $\begin{array}{l}14399(\mathrm{c}) \text {; main sequence compatible; } \\
\text { but not imbedded sequence } \\
n=11+21 j, j=68 \text { (Table } 1)\end{array}$ \\
\hline $\begin{array}{c}3,3,3 \\
(09)\end{array}$ & 7393 & 6043 & 4243 & 0 & 1767 & 0 & 589 & $\begin{array}{l}17679 \text { (c); main sequence blocks } \\
\text { any primes for } R=9(\text { Table } 1)\end{array}$ \\
\hline $\begin{array}{c}3,3,3 \\
(09)\end{array}$ & 1783 & 2843 & 3643 & 0 & 826 & 1 & 275 & 8269 (p); sequences compatible \\
\hline
\end{tabular}

Table 6(b). Structure of three prime sum with $R=9$

\section{Concluding comments}

\subsection{Numerical results}

A variety of sums for different numbers of arbitrary primes is listed in Tables 7(a) and 7(b):

\begin{tabular}{|c|c|c|c|c|c|c|c|c|c|c|c|}
\hline $\begin{array}{c}\text { No. } \\
\text { of } \boldsymbol{p}\end{array}$ & $\boldsymbol{p}_{\mathbf{1}}$ & $\boldsymbol{p}_{\mathbf{2}}$ & $\boldsymbol{p}_{\mathbf{3}}$ & $\boldsymbol{p}_{\mathbf{4}}$ & $\boldsymbol{p}_{\mathbf{5}}$ & $\boldsymbol{p}_{\mathbf{6}}$ & $\boldsymbol{p}_{\mathbf{7}}$ & $\boldsymbol{p}_{\mathbf{8}}$ & $\boldsymbol{p}_{\mathbf{9}}$ & $\sum p$ & $t_{0}=\left\lfloor\frac{n}{3}\right\rfloor$ \\
\hline \multirow{3}{*}{3} & 5227 & 3989 & 3121 & & & & & & & 12337 & 411 \\
\cline { 2 - 13 } & 2887 & 3701 & 5981 & & & & & & & 12569 & 418 \\
\cline { 2 - 13 } & 107 & 487 & 937 & & & & & & & 1531 & 51 \\
\hline \multirow{2}{*}{5} & 5869 & 677 & 1193 & 1747 & 5153 & & & & & 14639 & 487 \\
\cline { 2 - 12 } & 5987 & 4283 & 2917 & 433 & 683 & & & & & 14303 & 476 \\
\hline \multirow{2}{*}{7} & 2039 & 6229 & 7159 & 8053 & 6959 & 9349 & 641 & & & 40429 & 1347 \\
\cline { 2 - 12 } & 3623 & 4231 & 4877 & 5749 & 547 & 1447 & 1303 & & & 21777 & 725 \\
\hline \multirow{3}{*}{9} & 5569 & 6217 & 2643 & 7901 & 8581 & 9697 & 5927 & 3313 & 3659 & 53507 & 1783 \\
\cline { 2 - 11 } & 2953 & 3371 & 3833 & 4363 & 5197 & 5827 & 5861 & 971 & 2297 & 32989 & 1099 \\
\cline { 2 - 10 } & 2953 & 3371 & 3833 & 4363 & 5197 & 5827 & 5861 & 971 & 2297 & 34673 & 1155 \\
\hline
\end{tabular}

Table 7(a). Some prime sums 


\begin{tabular}{|c|c|c|c|c|}
\hline$\sum p$ & $\begin{array}{c}\mathrm{p} \\
\text { or c }\end{array}$ & $\begin{array}{c}\text { Main } \\
\text { sequence for } \\
n=3 t_{0}+\mathrm{a}\end{array}$ & $\begin{array}{c}\text { Imbedded } \\
\text { sequence } \\
\text { for } n=\end{array}$ & Comments \\
\hline 12337 & $\mathrm{c}$ & $a=0$ & $24+39 j$ & $\begin{array}{l}j=31 \text { only composites when } R=7 \text { for this } \\
\text { sequence (Table } 1 \text { ) }\end{array}$ \\
\hline 12569 & $\mathrm{p}$ & $a=2$ & $17+21 j$ & $j=59$ (imbedded sequence not in Table 1) \\
\hline 1531 & $\mathrm{p}$ & $a=0$ & $6+21 j$ & $j=7$ (imbedded sequence not in Table 1) \\
\hline 14639 & $\mathrm{p}$ & $a=2$ & $14+21 j$ & $j=69$ (imbedded sequence not in Table 1) \\
\hline 14303 & $\mathrm{p}$ & $a=2$ & $2+21 j$ & $j=68$ (imbedded sequence not in Table 1 ) \\
\hline 40429 & $\mathrm{p}$ & $a=1$ & $10+21 j$ & $j=192$ (imbedded sequence not in Table 1) \\
\hline 21777 & $\mathrm{c}$ & $a=2$ & & $\begin{array}{l}\text { Main sequence incompatible }- \text { invalid } f(t) \text { for } R \\
=7 \text {, cannot be prime }\end{array}$ \\
\hline 53507 & $\mathrm{p}$ & $a=1$ & $16+21 j$ & $j=254$ (imbedded sequence not in Table 1) \\
\hline 32989 & $\mathrm{c}$ & $a=1$ & $64+231 j$ & $\begin{array}{l}j=14 \text {; only composites when } R=9 \text { for this } \\
\text { sequence of } n \text { (Table } 1)\end{array}$ \\
\hline 34673 & $\mathrm{p}$ & $a=2$ & $2+21 j$ & $j=165 ;$ sequences compatible (not in Table 1$)$ \\
\hline
\end{tabular}

Table 7(b). Comments for some prime sums

\subsection{General results}

Table 1 will obviously be incomplete as larger and larger integers are considered, and new imbedded sequences are revealed. However, the advantage of knowing the structural characteristics of the prime sums remains the same. The principles apply irrespective of the numbers of primes which are summed or the size of the integers as illustrated in Tables 7 (a) and (b).

\section{References}

[1] Vassilev-Missana, M., \& Atanassov, K. T. (2004) Some Smarandache Problems. Phoenix, AZ: Hexis.

[2] Leyendekkers, J. V., \& Shannon, A. G. (2018) Prime sequences. Notes on Number Theory and Discrete Mathematics, 24 (3), 77-83.

[3] Shannon, A. G, \& Leyendekkers, J. V. (2018) The Fibonacci Numbers and Integer Structure. New York: Nova.

[4] Leyendekkers, J. V., \& Shannon, A. G. (2018) Structural sequences for primes using rightend-digits. Notes on Number Theory and Discrete Mathematics, 24 (2), 63-70. 